\title{
COMPOSITION WITH A TWO VARIABLE FUNCTION
}

\author{
Gil Guibert, François Loeser, and Michel Merle
}

\section{Introduction}

In [11] and [12], A. Némethi studied the Milnor fiber and monodromy zeta function of composed functions of the form $f\left(g_{1}, g_{2}\right)$ with $f$ a two variable polynomial and $g_{1}$ and $g_{2}$ polynomials with distinct sets of variables. The present paper addresses the question of proving similar results for the motivic Milnor fiber introduced by Denef and Loeser, cf. [1],[3],[10],[4]. In fact, Némethi later considered in [13] the more general situation of a composition $f \circ \mathbf{g}:(X, x) \rightarrow\left(\mathbb{C}^{2}, 0\right) \rightarrow(\mathbb{C}, 0)$, where $\mathbf{g}$ has a reasonable discriminant. Still later, Némethi and Steenbrink [14] proved similar results at the level of the Hodge spectrum [16],[17],[18], using the theory of mixed Hodge modules. In particular, they were able to compute, under mild assumptions, the Hodge spectrum of composed functions of the form $f\left(g_{1}, g_{2}\right)$ without assuming the variables in $g_{1}$ and $g_{2}$ are distinct. Their result involves the discriminant of the morphism $\mathbf{g}=\left(g_{1}, g_{2}\right)$. In a previous paper [7], we computed the motivic Milnor fiber for functions of the form $g_{1}+g_{2}^{\ell}$ when $\ell$ is large without assuming the variables in $g_{1}$ and $g_{2}$ are distinct. The corresponding result for the Hodge spectrum goes back to M. Saito [15] and is a special case of the results of Némethi and Steenbrink [14]. So, it seems very natural to search for a full motivic analogue of the results of [14]. At the present time, we are unable to realize this program and we have to limit ourself, as we already mentioned, to the case when $g_{1}$ and $g_{2}$ have no variable in common. Already extending our result to the case when one only assumes the discriminant of the morphism $\mathbf{g}$ is contained in the coordinate axes seems to require new ideas.

In this paper we consider a polynomial $f$ in $k[x, y]$ and we assume that $f(0, y)$ is non zero of degree $m$. We denote by $i_{p}$ the closed embedding into $\mathbb{A}_{k}^{2}$ of a point $p$ in $F_{0}=f^{-1}(0) \cap x^{-1}(0)$. We consider the motivic Milnor fiber $\mathcal{S}_{f}$ of the function $f: \mathbb{A}_{k}^{2} \longrightarrow \mathbb{A}_{k}^{1}$ whose restriction $i_{p}^{*} \mathcal{S}_{f}$ above $p$ is an element of the Grothendieck ring $\mathcal{M}_{\mathbb{G}_{m}}^{\mathbb{G}_{m}}$. We then reformulate Guibert's computation of the motivic Milnor fiber of germs of plane curve singularities [6] using generalized convolution operators of [8]. More precisely, we express it in terms of the tree $\tau(f, p)$ associated to $f$, depending on the given coordinate system $(x, y)$ on the affine plane $\mathbb{A}_{k}^{2}$. Let us recall the tree $\tau(f, p)$ is obtained by considering the Puiseux expansions of the roots of $f$ at $p$, cf. [9], [5]. To any so-called rupture vertex $v$ of this graph, we attach a weighted homogeneous polynomial $Q_{v, f}$ in $k[c, d]$. We have defined in [8] a generalized convolution by such

Received by the editors November 19, 2007. 
a polynomial. It is a morphism from $\mathcal{M}_{\mathbb{G}_{m} \times \mathbb{G}_{m}}^{\mathbb{G}_{m}}$ to $\mathcal{M}_{\mathbb{G}_{m}}^{\mathbb{G}_{m}}$, but can be extended to a morphism from $\mathcal{M}_{\mathbb{A}_{k}^{2} \times \mathbb{G}_{m}}^{\mathbb{G}_{m}}$ to $\mathcal{M}_{\mathbb{G}_{m}}^{\mathbb{G}_{m}}$. We denote by $\varpi_{j}$ the morphism $x \longmapsto x^{j}$ from $\mathbb{G}_{m}$ to $\mathbb{G}_{m}$ and by $m_{p}$ the order of $p$ as a root of $f(0, y)$. One can then reformulate Guibert's theorem as

$(*) \quad i_{p}^{*} \mathcal{S}_{f}=\left[\varpi_{m_{p}}: \mathbb{G}_{m} \longrightarrow \mathbb{G}_{m}\right]-\sum_{v} \Psi_{Q_{v, f}}\left(\left[\mathrm{Id}: \mathbb{A}_{k}^{1} \times \mathbb{G}_{m} \longrightarrow \mathbb{A}_{k}^{1} \times \mathbb{G}_{m}\right]\right)$

where the sum runs over the set of rupture vertices of $\tau(f, p)$.

For $1 \leq j \leq 2$, let $g_{j}: X_{j} \longrightarrow \mathbb{A}_{k}^{1}$ be a function on a smooth $k$-variety $X_{j}$. By composition with the projection, $g_{j}$ becomes a function on the product $X=X_{1} \times X_{2}$ and we write $\mathbf{g}$ for the map $g_{1} \times g_{2}: X \rightarrow \mathbb{A}_{k}^{2}$. The main result of this paper, Theorem 4.2 , gives a formula for $i^{*} \mathcal{S}_{f \circ \mathrm{g}}$, where $i$ denote the inclusion of $g_{1}^{-1}(0) \cap g_{2}^{-1}(0)$, similar to $(*)$, with $\left[\operatorname{Id}: \mathbb{A}_{k}^{1} \times \mathbb{G}_{m} \longrightarrow \mathbb{A}_{k}^{1} \times \mathbb{G}_{m}\right]$ replaced by a virtual object $A_{v}$. The virtual object $A_{v}$ is defined inductively in terms of the tree associated to $f$ at the origin of $\mathbb{A}_{k}^{2}$ and of $A_{v_{0}}$, where $v_{0}$ is the first (extended) rupture vertex of $\tau(f, p)$, and $A_{v_{0}}$ depends only on $\mathbf{g}$.

During the preparation of this paper, the authors have been partially supported by grant ANR-06-BLAN-0183.

\section{Preliminaries and combinatorial set up}

2.1. We fix an algebraically closed field $k$ of characteristic 0 . For a variety $X$ over $k$, we denote by $\mathcal{L}(X)$ and $\mathcal{L}_{n}(X)$ the spaces of arcs, resp. arcs mod $t^{n+1}$ as defined in [2]. As in [7], we denote by $\mathcal{M}_{X}$ the localisation of the Grothendieck ring of varieties over $X$ with respect to the class of the relative line. We shall also use the $\mathbb{G}_{m}$-equivariant variant $\mathcal{M}_{X \times \mathbb{G}_{m}^{p}}^{\mathbb{G}_{m}}$ defined in [8], which is generated by classes of objects $Y \rightarrow X \times \mathbb{G}_{m}^{p}$ endowed with a monomial $\mathbb{G}_{m}$-action.

Also, if $p$ is a closed point of $X$ we denote by $i_{p}$ the inclusion $i_{p}: p \rightarrow X$ and by $i_{p}^{*}$ the corresponding pullback morphism at the level of rings $\mathcal{M}$.

2.2. Let us start by recalling some basic constructions introduced by Denef and Loeser in [1], [4] and [3].

Let $X$ be a smooth variety over $k$ of pure dimension $d$ and $g: X \rightarrow \mathbb{A}_{k}^{1}$. We set $X_{0}(g)$ for the zero locus of $g$, and consider, for $n \geq 1$, the variety

$$
\mathcal{X}_{n}(g):=\left\{\varphi \in \mathcal{L}_{n}(X) \mid \operatorname{ord}_{t} g(\varphi)=n\right\} .
$$

Note that $\mathcal{X}_{n}(g)$ is invariant by the $\mathbb{G}_{m}$-action on $\mathcal{L}_{n}(X)$. Furthermore $g$ induces a morphism $g_{n}: \mathcal{X}_{n}(g) \rightarrow \mathbb{G}_{m}$, assigning to a point $\varphi$ in $\mathcal{L}_{n}(X)$ the coefficient ac $(g(\varphi))$ of $t^{n}$ in $g(\varphi)$, which we shall also denote by $\operatorname{ac}(g)(\varphi)$. This morphism is homogeneous of weight $n$ with respect to the $\mathbb{G}_{m}$-action on $\mathcal{X}_{n}(g)$ since $g_{n}(a \cdot \varphi)=a^{n} g_{n}(\varphi)$, so we can consider the class $\left[\mathcal{X}_{n}(g)\right]$ of $\mathcal{X}_{n}(g)$ in $\mathcal{M}_{X_{0}(g) \times \mathbb{G}_{m}}^{\mathbb{G}_{m}}$.

We now consider the motivic zeta function

$$
Z_{g}(T):=\sum_{n \geq 1}\left[\mathcal{X}_{n}(g)\right] \mathbb{L}^{-n d} T^{n}
$$

in $\mathcal{M}_{X_{0}(g) \times \mathbb{G}_{m}}^{\mathbb{G}_{m}}[[T]]$. Note that $Z_{g}=0$ if $g=0$ on $X$. 
Denef and Loeser showed in [1] and [3] (see also [4]) that $Z_{g}(T)$ is a rational series by giving a formula for $Z_{g}(T)$ in terms of a resolution of $f$. They also showed that one can consider $\lim _{T \mapsto \infty} Z_{g}(T)$ in $\mathcal{M}_{X_{0}(g) \times \mathbb{G}_{m}}^{\mathbb{G}_{m}}$ and they define the motivic Milnor fiber of $g$ as

$$
\mathcal{S}_{g}:=-\lim _{T \mapsto \infty} Z_{g}(T)
$$

2.3. In this subsection we do not assume $X$ to be smooth. For technical reasons we shall use in the present paper the following innocuous variant of $\mathcal{M}_{X \times \mathbb{G}_{m}^{p}}^{\mathbb{G}_{m}}$ : replacing everywhere in the definition the first $\mathbb{G}_{m}$-factor endowed with the $\mathbb{G}_{m}$-action by multiplicative translation $\lambda \cdot x=\lambda x$ by $\mathbb{A}_{k}^{1}$ with "the same" $\mathbb{G}_{m}$-action one gets a ring $\mathcal{M}_{X \times \mathbb{A}_{k}^{1} \times \mathbb{G}_{m}^{p-1}}^{\mathbb{G}_{m}}$ generated by classes of objects $Y \rightarrow X \times \mathbb{A}_{k}^{1} \times \mathbb{G}_{m}^{p-1}$ endowed with a monomial $\mathbb{G}_{m}$-action.

If $Q$ is a quasihomogeneous polynomial in $p$ variables, we defined in [8] a convolution operator

$$
\Psi_{Q}: \mathcal{M}_{X \times \mathbb{G}_{m}^{p}}^{\mathbb{G}_{m}} \longrightarrow \mathcal{M}_{X \times \mathbb{G}_{m}}^{\mathbb{G}_{m}}
$$

In this paper we shall use the slight variant, still denoted by $\Psi_{Q}$, which is obtained with the same definition, replacing $\mathbb{G}_{m}^{p}$ by $\mathbb{A}_{k}^{1} \times \mathbb{G}_{m}^{p-1}$,

$$
\Psi_{Q}: \mathcal{M}_{X \times \mathbb{A}_{k}^{1} \times \mathbb{G}_{m}^{p-1}}^{\mathbb{G}_{m}} \longrightarrow \mathcal{M}_{X \times \mathbb{G}_{m}}^{\mathbb{G}_{m}}
$$

In fact, such constructions carry over for any toric variety with torus $\mathbb{G}_{m}^{p}$, not only for $\mathbb{A}_{k}^{1} \times \mathbb{G}_{m}^{p-1}$.

2.4. Fix a positive integer $N$ and consider the ring of fractional power series $k\left[\left[x^{\frac{1}{N}}\right]\right]$. Given a positive rational number $r$ we denote by $I_{\geq r}$ the ideal of power series of order at least $r$ in $k\left[\left[x^{\frac{1}{N}}\right]\right]$. We call the quotient $k\left[\left[x^{\frac{1}{N}}\right]\right] / I_{\geq r}$ the ring of $r$-truncated fractional power series.

To a $r$-truncated fractional power series $y$ one assigns a labelled rooted real metric tree $\tau_{r}(y)$ in the following way. The total space $\tau_{r}(y)$ is the half-open interval $[0, r)$ and its vertices are the positive exponents with non zero coefficients of the expansion of $y$ in powers of $x$ together with the origin which is the root. We define the height of a vertex to be its distance to the root. We label each vertex by the coefficient of the corresponding term (this coefficient is non-zero for all vertices except maybe for the root). The vertices are ordered by the height. Starting above a vertex there is only one edge. This edge ends with the next vertex if there is one and remains open above the last vertex. We label each edge by 0 and we say $\tau_{r}(y)$ is of height $r$. We denote by $\left|\tau_{r}(y)\right|$ the underlying unlabelled tree. Notice that we can see the labels as degree 1 polynomials of $k[X]$ (or cycles in $\mathbb{A}_{k}^{1}$ ), that is, $X$ for an edge and $X-a$ for a vertex labelled by $a$.

If now $y$ is a power series in $k\left[\left[x^{\frac{1}{N}}\right]\right]$, we denote by $\tau_{r}(y)$ the height $r$ tree associated to its truncation at order $r$. Thus, for $r<r^{\prime}, \tau_{r}(y)$ is obtained from $\tau_{r^{\prime}}(y)$ by truncating up to height $r$. We denote by $\tau(y)$ the inductive limit of the system $\left(\tau_{r}(y)\right)_{r \in \mathbb{Q}}$ and call it the tree associated to the power series $y$. 
2.5. We consider a two variable polynomial $f$ in $k[x, y]$. We assume that $f(0, y)$ is non zero of degree $m$ and we consider the $m$ Newton-Puiseux expansions $y_{i}, 1 \leq i \leq m$, associated to $f$ at the points of $f^{-1}(0) \cap x^{-1}(0)$. There exists an integer $N$ such that these roots are elements of the ring of fractional power series $k\left[\left[x^{\frac{1}{N}}\right]\right]$, namely, they are the roots of the polynomial $f$ in $k\left[\left[x^{\frac{1}{N}}\right]\right]$.

Fix a positive rational number $r$. We denote by $\bigcup_{i=1}^{m} \tau_{r}\left(y_{i}\right)$ the labelled rooted real metric tree which is obtained as follows. In the disjoint union of the trees $\coprod_{i=1}^{m}\left|\tau_{r}\left(y_{i}\right)\right|$ we identify two vertices (resp. two edges) if they have same height and same label. If $v$ is a vertex (resp. an edge) shared by trees $\tau_{r}\left(y_{i}\right)$ for $i$ in $J$, then its label on the union is the $|J|$-th power of its label on any of the $\tau_{r}\left(y_{i}\right), i$ in $J$.

Since the group of $N$-roots of unity acts on the $r$-truncated expansions $\left(y_{1}, \ldots, y_{m}\right)$, it also acts on $\left|\bigcup_{i=1}^{m} \tau_{r}\left(y_{i}\right)\right|$. We denote by $\left|\tau_{r}(f)\right|$ the separated quotient and by $\pi$ : $\left|\bigcup_{i=1}^{m} \tau_{r}\left(y_{i}\right)\right| \rightarrow\left|\tau_{r}(f)\right|$ the quotient morphism. Note that the connected components of $\left|\tau_{r}(f)\right|$ are in natural bijection with points of $f^{-1}(0) \cap x^{-1}(0)$. For any such point $p$, we denote by $|\tau(f, p)|$ the corresponding connected component which is naturally endowed with the structure of a rooted real metric tree. We attach labels to the vertices and edges of $\left|\tau_{r}(f)\right|$ in the following way:

- If $e$ is an edge of $\left|\tau_{r}(f)\right|$, the label attached to $e$ is the label on any element of $\pi^{-1}(e)$. It is a power of $X$ in $k[X]$ and we denote it by $P_{e, f}$. We will call degree of the edge $e$ the degree of $P_{e, f}$.

- If $v$ is a vertex of $\left|\tau_{r}(f)\right|$, the label on $v$ is the product of the labels on $\pi^{-1}(v)$. We denote it by $P_{v, f}$. We will call degree of the vertex $v$ the degree of $P_{v, f}$. Notice that the degree of a vertex $v$ is equal to the degree of the edge $e$ which ends in $v$.

For $r<r^{\prime}$, the graph $\tau_{r}(f)$ is the truncation of $\tau_{r^{\prime}}(f)$ at height $r$. The graph of contacts $\tau(f)$ defined by $f$ along $f^{-1}(0) \cap x^{-1}(0)$ is the inductive limit of the graphs $\tau_{r}(f), r \in \mathbb{Q}$, cf. [9], [5]. We say that a vertex $v$ of $\tau(f)$ is a rupture vertex if the set of zeroes of $P_{v, f}$ contains at least two points in $\mathbb{A}_{k}^{1}$. We define the augmented set of rupture vertices of the tree $\tau(f, p)$ as the set of rupture vertices of $\tau(f, p)$ together with the vertex of minimal non zero height on $\tau(f, p)$.

We fix from now on a point $p$ which will be assumed for simplicity to be the origin in $\mathbb{A}_{k}^{2}$. For any $\operatorname{arc} \varphi$ in $\mathcal{L}\left(\mathbb{A}_{k}^{2}\right)$ such that $\varphi(0)=p$ and $x(\varphi) \neq 0$, there exist power series $\omega$ in $k[[t]]$ and $\sum_{j} b_{j} \omega^{j}$ in $k[[\omega]]$, and an integer $M$ such that $\operatorname{gcd}\left(M,\left\{j \mid b_{j} \neq 0\right\}\right)=1$ and

Hence

$$
\begin{aligned}
& x(\varphi(t))=\omega(t)^{M} \\
& y(\varphi(t))=\sum_{j} b_{j} \omega(t)^{j} .
\end{aligned}
$$

$$
y(\varphi(t))=\sum_{j} b_{j}\left(x(\varphi(t))^{\frac{j}{M}}\right.
$$

is a fractional power series in $x(\varphi(t))$. We consider the tree $\tau(y)$ with

$$
y(x):=\sum_{j} b_{j} x^{\frac{j}{M}}
$$

in $k\left[\left[x^{\frac{1}{M}}\right]\right]$.

The power series $\omega$ is defined up to an $M$-rooth of unity so that the $b_{j}$ 's are defined up to a factor $\zeta^{j}$ with $\zeta$ an $M$-root of unity. Two different choices lead to trees in 
the same $\mu_{N}$-orbit. This orbit is denoted by $\tau(\varphi)$. Notice that $\tau(\varphi)$, as well as $\tau(f)$, depends on the system of coordinates $(x, y)$.

2.6. Definition. Consider $\varphi$ in $\mathcal{L}\left(\mathbb{A}_{k}^{2}\right)$ and $f$ in $k[x, y]$ as before. The order of contact of $\varphi$ with $f$ is the maximum number $s$ in $\mathbb{Q} \cup\{\infty\}$ such that $\tau_{s}(\varphi)$ is included in $\tau_{s}(f)$ (it is infinite if and only if $f(\varphi)=0$ ). The contact of $\varphi$ with $f$ is the tree $\tau_{r}(\varphi)$ where $r$ is the order of contact of $\varphi$ with $f$.

2.7. From now on the polynomial $f$ is fixed in $k[x, y]$ and we denote by $m$ the degree of $f(0, y)$. For a positive rational number $r$, by a contact $\tau$ of order $r$, we mean a subtree of $\tau(f, p)$ which is isomorphic to $[0, r)$. In particular $\tau$ is rooted at $p$ and its closure in $\tau(f, p)$ contains a unique point of height $r$, not necessarily a vertex of $\tau(f, p)$, which completely determines $\tau$. To such a contact $\tau$ we assign a polynomial $P_{\tau, f}$ in the following way. The last and (semi)open edge of $\tau$ is contained in a unique edge $e$ of $\tau(f, p)$.

- If $e$ ends at a vertex $v$ at height $r$ of $\tau(f, p)$ (in this case we say that $\tau$ ends at the vertex $v$ ), we will set $P_{\tau, f}=P_{\tau, v}$.

- Otherwise, (in that case we say that $\tau$ ends at the edge $e$ ) we set $P_{\tau, f}=P_{\tau, e}$.

By definition of contact, there is an integer $M$ and a polynomial $y_{\tau}$ in $k[\omega]$, of degree strictly smaller than $r M$, both depending only on $\tau$, such that for any $\operatorname{arc} \varphi$ of contact $\tau$ with $f$, there exists a series $\omega$ in $k[[t]], \operatorname{ord}_{t}(\omega)=\ell$, such that

$$
\begin{aligned}
& x(\varphi(t))=\omega(t)^{M} \\
& y(\varphi(t))=y_{\tau}(\omega(t)) \quad\left[\bmod \left(t^{\lceil r M \ell\rceil}\right)\right] .
\end{aligned}
$$

For an $\operatorname{arc} \varphi$ of contact $\tau$ with $f$, the quotient $\operatorname{ord}_{t}(f(\varphi)) / \ell$ is an integer and depends only on $\tau$. We denote it by $\nu(\tau)$. One always has the inequality $\nu(\tau) \geq M r$.

The tree $\tau(f, p)$ is built from the Puiseux expansions of the $m$ roots of $f(x, y)$ in the ring of fractional power series $\bigcup_{N} k\left[\left[x^{1 / N}\right]\right]$. Conversely, to any finite subtree $\varsigma$ of $\tau(f, p)$, we can associate a polynomial $f_{\varsigma}$ in $k[x, y]$ which is the minimal polynomial of the $m$ Puiseux expansions restricted to $\varsigma$. Considering the tree associated to the polynomial $f_{\varsigma}$, we get a tree $\tau\left(f_{\varsigma}, p\right)$ which is an infinite tree with a finite number of vertices. The intersection of $\tau\left(f_{\varsigma}, p\right)$ with $\tau(f, p)$ contains $\varsigma$. As an example, we can consider the tree $\tau_{r}$ obtained from $\tau(f, p)$ by truncation at height $r$. We will denote by $\overline{\tau_{r}}$ the tree $\tau\left(f_{\tau_{r}}, p\right)$.

\section{Guibert's theorem revisited}

3.1. We consider the following set of arcs:

$$
\mathcal{X}_{\tau, \ell}:=\left\{\varphi \in \mathcal{L}_{\bar{\nu}(\tau) \ell}\left(\mathbb{A}_{k}^{2}\right) \mid \varphi \text { has contact } \tau \text { with } f, \operatorname{ord}_{t} x(\varphi)=M \ell\right\} .
$$

where $\bar{\nu}(\tau)$ is the maximum of the integers $\nu(\tau)$ and $M$.

We denote by $Q_{\tau, f}$ the function $(\omega, c) \mapsto \omega^{\nu(\tau)} P_{\tau, f}\left(\omega^{-M r} c\right)$. One should note that $Q_{\tau, f}$ is a polynomial in $k[c, \omega]$, even if $M r$ may not be an integer.

3.2. Lemma. Consider a contact $\tau$ and an integer $\ell$ and denote by $N(\tau, \ell)$ the integer $2 \bar{\nu}(\tau) \ell-M \ell-\lfloor M r \ell\rfloor$. For any arc $\varphi$ in $\mathcal{X}_{\tau, \ell}$, there exist two series $\omega$ and $\varepsilon$ in $k[t] / t^{\bar{\nu}(\tau) \ell+1}$ such that

(1) $\operatorname{ord}_{t}(\omega)=\ell, x(\varphi)=\omega^{M}$ 
(2) $\operatorname{ord}_{t}(\varepsilon) \geq M r \ell$ (resp. $=M r \ell$ if $\tau$ ends in an edge), $y(\varphi)=y_{\tau}(\omega)+\varepsilon$. The mapping $(\varepsilon, \omega) \longmapsto\left(\omega^{M}, y_{\tau}(\omega)+\varepsilon\right)$ induces an isomorphism

$$
\Phi:\left(\left(\mathbb{A}_{k}^{1} \times \mathbb{G}_{m}\right) \backslash Q_{\tau, f}^{-1}(0)\right) \times \mathbb{A}_{k}^{N(\tau, \ell)} \longrightarrow \mathcal{X}_{\tau, \ell}
$$

sending $\left(c, \omega_{\ell}, a\right)$ to

$$
\left(t^{\ell M}\left(\omega_{\ell}+\sum_{k=1}^{\ell(\bar{\nu}(\tau)-M)} a_{k} t^{k}\right)^{M}\left[t^{\ell \bar{\nu}(\tau)+1}\right], y_{\tau}(\omega)+c t^{M r \ell}+\sum_{\ell(\bar{\nu}(\tau)-M)<k \leq \ell \bar{\nu}(\tau)} a_{k} t^{k}\left[t^{\ell \bar{\nu}(\tau)+1}\right]\right) .
$$

Via the isomorphism $\Phi$, the angular coefficient $\operatorname{ac}(f(\varphi))$ is given, up to a non-zero constant, by the following formula:

$$
\operatorname{ac}(f(\varphi)) \sim \omega_{\ell}^{\nu(\tau)} P_{\tau, f}\left(\omega_{\ell}^{-M r} c\right)=Q_{\tau, f}\left(c, \omega_{\ell}\right) .
$$

For the $\mathbb{G}_{m}$-action $\sigma$ on $\left(\mathbb{A}_{k}^{1} \times \mathbb{G}_{m}\right)$ given by $\sigma(\lambda) \cdot\left(c, \omega_{\ell}\right)=\left(\lambda^{M r \ell} c, \lambda^{\ell} \omega_{\ell}\right)$, the polynomial $Q_{\tau, f}$ is homogeneous of degree $\nu(\tau) \ell$.

Proof. We did already notice that the map $\Phi$ is surjective. Conversely $\omega$ is determined by $x(\varphi)$ up to a $M$-th root of unity, and uniquely determined by $x(\varphi)$ and $y(\varphi)$ for the $g c d$ of $M$ and exponents of non zero terms in $y_{\tau}(\omega)$ is equal to 1 .

3.3. On the constructible set $\mathcal{X}_{\tau, \ell}$, via the isomorphism $\Phi$, we have a morphism to $\mathbb{A}_{k}^{1} \times \mathbb{G}_{m}$ induced by the first projection from $\left(\mathbb{A}_{k}^{1} \times \mathbb{G}_{m}\right) \times \mathbb{A}_{k}^{N(\tau, \ell)}$. The constructible set $\mathcal{X}_{\tau, \ell}$ defines a class in $\mathcal{M}_{\mathbb{A}^{1} \times \mathbb{G}_{m}}^{\mathbb{G}_{m}}$ we denote by $\left[\mathcal{X}_{\tau, \ell}\right]$. On the other hand, the function ac $(f)$ induces a $\mathbb{G}_{m}$-equivariant morphism from $\mathcal{X}_{\tau, \ell}$ to $\mathbb{G}_{m}$, hence defines a class in $\mathcal{M}_{\mathbb{G}_{m}}^{\mathbb{G}_{m}}$ we denote by $\left[\mathcal{X}_{\tau, \ell}(f)\right]$. By Lemma 3.2 , the morphism $\mathcal{X}_{\tau, \ell} \rightarrow \mathbb{G}_{m}$ is equal to the composition of the morphism $\mathcal{X}_{\tau, \ell} \rightarrow \mathbb{A}_{k}^{1} \times \mathbb{G}_{m}$ with $Q_{\tau, f}$.

3.4. For any rupture vertex $v$, there is only one contact ending in $v$ that we denote by $\tau_{v}$. We set $Q_{v, f}:=Q_{\tau_{v}, f}$.

We are now in position to restate Guibert's theorem [6] in the following form:

3.5. Theorem (Guibert). With the above notation, the following holds:

$$
i_{p}^{*} \mathcal{S}_{f}=\left[\varpi_{m_{p}}: \mathbb{G}_{m} \longrightarrow \mathbb{G}_{m}\right]-\sum_{v} \Psi_{Q_{v, f}}\left(\left[\mathrm{Id}: \mathbb{A}_{k}^{1} \times \mathbb{G}_{m} \longrightarrow \mathbb{A}_{k}^{1} \times \mathbb{G}_{m}\right]\right)
$$

where the second sum runs over the rupture vertices of $\tau(f)$ above $p$.

Proof. Note that for a two variable quasihomogeneous polynomial $Q$

$$
\begin{aligned}
\Psi_{Q}\left(\left[\mathrm{Id}: \mathbb{A}_{k}^{1} \times \mathbb{G}_{m}\right.\right. & \left.\left.\longrightarrow \mathbb{A}_{k}^{1} \times \mathbb{G}_{m}\right]\right)= \\
& -\left[Q:\left(\mathbb{A}_{k}^{1} \times \mathbb{G}_{m}\right) \backslash Q^{-1}(0) \longrightarrow \mathbb{G}_{m}\right]+\mathcal{S}_{Q}\left(\left[\mathbb{A}_{k}^{1} \times \mathbb{G}_{m}\right]\right)
\end{aligned}
$$

where $\mathcal{S}_{Q}$ is defined as in [7], [8]. We denote by $\pi_{E}$ the morphism $(a, b) \longmapsto a^{E}$ from $\mathbb{G}_{m} \times \mathbb{G}_{m}$ to $\mathbb{G}_{m}$. When the zeroes of $Q$ are a disjoint union of one dimensional $\mathbb{G}_{m}$-orbits, $\mathcal{S}_{Q}\left(\mathbb{A}_{k}^{1} \times \mathbb{G}_{m}\right)$ decomposes into a sum

$$
\mathcal{S}_{Q}\left(\mathbb{A}_{k}^{1} \times \mathbb{G}_{m}\right)=-\sum_{i}\left[\pi_{E_{i}}: \mathbb{G}_{m} \times \mathbb{G}_{m} \longrightarrow \mathbb{G}_{m}\right]
$$


where $E_{i}$ is the multiplicity of $Q$ along the $i$-th component of $Q^{-1}(0)$. If $v$ is a rupture vertex we consider the following zeta function:

$$
Z_{f}^{v}(T):=\sum_{\ell \geq 1} \sum_{\tau}\left[\mathcal{X}_{\tau, \ell}(f)\right] \mathbb{L}^{-2 \nu(\tau) \ell} T^{\nu(\tau) \ell}
$$

where the second sum is extended to the set of contacts $\tau$ which contain $\tau_{v}$ and do not contain or end in any successor of $v$ in the set of rupture vertices. From [6] (3.3) and (5.2), we deduce that $Z_{f}^{v}(T)$ has a limit $-\mathcal{S}_{f}^{v}$ in the Grothendieck ring $\mathcal{M}_{\mathbb{G}_{m}}^{\mathbb{G}_{m}}$ when $T$ goes to infinity, which is given by the formula

$$
\mathcal{S}_{f}^{v}=-\Psi_{Q_{v, f}}\left(\left[\mathrm{Id}: \mathbb{A}_{k}^{1} \times \mathbb{G}_{m} \longrightarrow \mathbb{A}_{k}^{1} \times \mathbb{G}_{m}\right]\right) .
$$

We consider now the series

$$
Z_{f}^{p}(T):=\sum_{\ell \geq 1} \sum_{\tau}\left[\mathcal{X}_{\tau, \ell}(f)\right] \mathbb{L}^{-2 \nu(\tau) \ell} T^{\nu(\tau) \ell}
$$

where the second sum is extended to the set of contacts $\tau$ starting from the root corresponding to $p$, which do not contain or end in any rupture vertex. Again by [6], loc. cit., $Z_{f}^{p}(T)$ has limit

$$
-\left[\varpi_{m_{p}}: \mathbb{G}_{m} \longrightarrow \mathbb{G}_{m}\right]
$$

when $T$ goes to infinity. The restriction $i_{p}^{*} \mathcal{S}_{f}$ is the limit as $T \mapsto \infty$ of $-i_{p}^{*} Z_{f}(T)$ which decomposes into

$$
-i_{p}^{*} Z_{f}(T)=-Z_{f}^{p}(T)-\sum_{v} Z_{f}^{v}(T)
$$

where the sum extends to all the rupture vertices of $\tau(f)$ above $p$. The result follows.

\section{Composition with a morphism}

4.1. For $1 \leq j \leq 2$, let $g_{j}: X_{j} \longrightarrow \mathbb{A}_{k}^{1}$ be a function on a smooth $k$-variety $X_{j}$. By composition with the projection, $g_{j}$ becomes a function on the product $X=X_{1} \times X_{2}$. We write $d_{j}$ for the dimension of $X_{j}, j$ from 1 to 2 , and $d$ for $d_{1}+d_{2}$. Define $\mathbf{g}$ as the map $g_{1} \times g_{2}$ on $X$ and $G$ as the product $G=g_{1} g_{2}$. For any subvariety $Z$ of the set $X_{0}(G)$ containing $X_{0}(\mathbf{g}):=g_{1}^{-1}(0) \cap g_{2}^{-1}(0)$, we denote by $i$ the closed immersion of $X_{0}(\mathrm{~g})$ in $Z$.

As in section 2 , we denote by $f$ a two variable polynomial, we assume that $f(0, y)$ is a nonzero polynomial, we denote by $p$ the origin and will denote by $m_{p}$ the order of 0 as a root of $f(0, y)$. We consider the augmented set of rupture vertices of the tree $\tau(f, p)$, namely the set of rupture vertices together with the vertex of minimal non zero height on $\tau(f, p)$. Denote that vertex by $v_{0}$ and consider its associated polynomial $Q_{v_{0}}$. We denote by $\mathcal{S}_{g_{2}}^{\prime}$ the element in $\mathcal{M}_{X_{0}\left(g_{2}\right) \times \mathbb{A}_{k}^{1}}^{\mathbb{G}_{m}}$ which is the "disjoint sum" of $\mathcal{S}_{g_{2}}$ in $\mathcal{M}_{X_{0}\left(g_{2}\right) \times \mathbb{G}_{m}}^{\mathbb{G}_{m}}$ and $X_{0}\left(g_{2}\right)$ in $\mathcal{M}_{X_{0}\left(g_{2}\right)}$. We set $A_{v_{0}}:=\mathcal{S}_{g_{2}}^{\prime} \otimes \mathcal{S}_{g_{1}}$, considered as an element in $\mathcal{M}_{X_{0}(\mathbf{g}) \times\left(\mathbb{A}_{k}^{1} \times \mathbb{G}_{m}\right)}^{\mathbb{G}_{m}}$. For any rupture vertex $v$ of the tree $\tau(f, p)$, we denote by $a(v)$ the predecessor of $v$ in the augmented set of rupture vertices and we define by induction a virtual variety $A_{v}$ in $\mathcal{M}_{X_{0}(\mathbf{g}) \times \mathbb{A}_{k}^{1} \times \mathbb{G}_{m}}^{\mathbb{G}_{m}}$. We assume we are given a virtual variety $A_{a(v)}$ in $\mathcal{M}_{X_{0}(\mathbf{g}) \times \mathbb{A}_{k}^{1} \times \mathbb{G}_{m}}^{\mathbb{G}_{m}}$ whose restriction over $X_{0}(\mathrm{~g}) \times \mathbb{G}_{m} \times \mathbb{G}_{m}$ is diagonally monomial in the sense of [7] (2.3), or more precisely whose $\mathbb{G}_{m}$-action is 
diagonally induced from a diagonally monomial $\mathbb{G}_{m}^{2}$-action in the sense of [7]. To any successor of $a(v)$ corresponds a factor of the polynomial $Q_{a(v)}$. Denote by $Q_{a(v)}^{v}$ the factor associated to $v$. Notice that $\left(Q_{a(v)}^{v}\right)^{-1}(0)$ is a smooth subvariety in $\mathbb{G}_{m} \times \mathbb{G}_{m}$ equivariant under a diagonal $\mathbb{G}_{m}$-action and that the second projection $\mathrm{pr}_{2}$ of the product $\mathbb{A}_{k}^{1} \times \mathbb{G}_{m}$ induces an homogeneous fibration from $\left(Q_{a(v)}^{v}\right)^{-1}(0)$ to $\mathbb{G}_{m}$. We denote by $B_{v}$ the restriction of $A_{a(v)}$ above $\left(Q_{a(v)}^{v}\right)^{-1}(0)$. The external product of the identity of the affine line $\mathbb{A}_{k}^{1}$ by the induced map $\mathrm{pr}_{2}: B_{v} \longrightarrow \mathbb{G}_{m}$ defines an element $A_{v}$ in $\mathcal{M}_{X_{0}(\mathbf{g}) \times\left(\mathbb{A}_{k}^{1} \times \mathbb{G}_{m}\right)}^{\mathbb{G}_{m}}$ which is diagonally monomial when restricted to $X_{0}(\mathrm{~g}) \times \mathbb{G}_{m} \times \mathbb{G}_{m}$.

4.2. Theorem. With the previous notations and hypotheses, the following formula holds

$$
i^{*} \mathcal{S}_{f \circ \mathrm{g}}=\mathcal{S}_{\left(g_{2}\right)^{m_{p}}}\left(X_{0}\left(g_{1}\right)\right)-\sum_{v} \Psi_{Q_{v}}\left(A_{v}\right),
$$

where the sum is runs over the augmented set of rupture vertices of the tree $\tau(f, p)$.

Proof. We first reduce to the case where $\tau(f, p)$ has only a finite number of vertices.

4.3. Lemma. There exists a rational number $\gamma$ such that, for any $r$ greater than $\gamma$,

$$
i^{*} \mathcal{S}_{f \circ \mathrm{g}}=i^{*} \mathcal{S}_{f_{\tau_{r}} \circ \mathrm{g}} .
$$

Proof. Consider a rupture vertex $v$. The quotient $\operatorname{ord}_{t}(f \circ \mathbf{g}(\varphi)) / \operatorname{ord}_{t}\left(g_{1}(\varphi)\right)$ is an affine function of $r$ whenever $\tau$ contains $\tau_{v}$ and does not contain or end in any rupture vertex greater than $v$. Hence the quotient $\operatorname{ord}_{t}(f \circ \mathbf{g}(\varphi)) / \operatorname{ord}_{t}\left(g_{1}(\varphi)\right)$ is a function on the tree $\tau(f)$, the restriction of which on each semi-open branch joining two consecutive rupture vertices (resp. on each infinite branch above a rupture vertex) is an increasing affine function of the height.

We consider the following set of arcs

$$
\mathcal{X}_{n_{1}, n_{2}}(x \circ \mathbf{g}, f \circ \mathbf{g}):=\left\{\varphi \in \mathcal{L}_{n_{1}+n_{2}}(X) \mid \operatorname{ord}_{t} x \circ \mathbf{g}=n_{1}, \operatorname{ord}_{t} f \circ \mathbf{g}=n_{2}\right\} .
$$

For $\gamma$ large enough, the zeta function

$$
Z_{x \circ \mathbf{g}, f \circ \mathbf{g}}^{\gamma}(T)=\sum_{n_{2} \geq \gamma n_{1}}\left[\mathcal{X}_{n_{1}, n_{2}}(x \circ \mathbf{g}, f \circ \mathbf{g})\right] \mathbb{L}^{-\left(n_{1}+n_{2}\right) d} T^{n_{2}}
$$

goes to zero as $T$ goes to infinity, cf. [7]. The lemma follows.

To prove the theorem, it is enough to consider the case when the tree $\tau(f, p)$ has a finite number of vertices. The proof goes by induction on the number of vertices of the tree $\tau(f, p)$. Certainly the result holds if there is no vertex. Assume first the tree has only one vertex $v_{0}$. The formula is then a particular case of the main formula in [8]. Assume now we have at least two vertices. Choose a maximal vertex $v$ for the height function on $\tau(f, p)$ and consider the subtree $\tau^{-}$obtained from $\tau(f, p)$ by deleting the vertex $v$. Denote by $a(v)$ the predecessor of $v$ on $\tau(f, p)$ and by $f^{-}$the polynomial associated to $\tau^{-}$, cf. 2.7 .

Consider an $\operatorname{arc} \varphi$ in $\mathbb{A}_{k}^{2}$ with origin $p$. Then one of the following two statements holds: 
- The contact of $\varphi$ with $f$ does not contain $\tau_{v}$. Then $\operatorname{ord}_{t}(f(\varphi))=\operatorname{ord}_{t}\left(f^{-}(\varphi)\right)$ and $\operatorname{ac}(f(\varphi))=\operatorname{ac}\left(f^{-}(\varphi)\right)$.

- The contact of $\varphi$ with $f$ contains $\tau_{v}$.

According to these two different cases, we can split the zeta function $Z_{f \circ g}$ in two pieces, namely

$$
Z_{f \circ g}=Z_{<v}+Z_{\geq v}
$$

Similarly, the zeta function $Z_{f-\circ}$ decomposes in

$$
Z_{f^{-} \circ \mathrm{g}}=Z_{<v}^{-}+Z_{\geq v}^{-} .
$$

We noticed that $Z_{<v}^{-}=Z_{<v}$, hence we get

$$
Z_{f \circ \mathrm{g}}-Z_{f-\mathrm{og}}=Z_{\geq v}-Z_{\geq v}^{-} .
$$

In section 3.3, for any contact $\tau$ and integer $\ell$, we have considered a set $\mathcal{X}_{\tau, \ell}$ associated to a polynomial $f$. Similarly we have a set $\mathcal{X}_{\tau, \ell}^{-}$associated to $f^{-}$. These two sets map to $\mathbb{A}_{k}^{1} \times \mathbb{G}_{m}$. Consider now the inverse image by $\mathbf{g}$ of $\mathcal{X}_{\tau, \ell}$ (resp. $\mathcal{X}_{\tau, \ell}^{-}$) and denote it by $\mathcal{X}_{\tau, \ell}(\mathbf{g})\left(\right.$ resp. $\left.\mathcal{X}_{\tau, \ell}^{-}(\mathbf{g})\right)$. We assume, by induction, that the motivic nearby cycles of $f^{-}$have the given form and that for any contact $\tau$ greater than $\tau_{a(v)}$ the set $\mathcal{X}_{\tau, \ell}^{-}(\mathbf{g})$ is a piecewise affine bundle on $X_{0}(\mathbf{g}) \times \mathbb{G}_{m} \times B_{a(v)}$.

An $\operatorname{arc} \mathbf{g} \circ \varphi$ in $\mathcal{L}_{\nu(\tau) \ell}\left(\mathbb{A}_{k}^{2}\right)$ having contact $\tau$ with $f^{-}$has contact $\tau_{v}$ with $f$ if and only if $\tau=\tau_{v}$ and $Q_{a(v)}^{v}$ does not vanish on $\varphi$ or if $\tau$ contains strictly $\tau_{v}$. In that case $\varphi$ maps to $\{0\} \times B_{a(v)}$. Hence the set $\mathcal{X}_{\tau_{v}, \ell}(\mathbf{g})$ is a disjoint union of piecewise affine bundles on $X_{0}(\mathbf{g}) \times\left(\left(\mathbb{A}_{k}^{1} \times B_{a(v)}\right) \backslash B_{v}\right)$ and the function ac $(f \circ \mathbf{g})$ is given by the composition of the canonical map with $Q_{v}$.

An arc $\mathbf{g} \circ \varphi$ in $\mathcal{L}_{\nu(\tau) \ell}\left(\mathbb{A}_{k}^{2}\right)$ has contact greater than $\tau_{v}$ with $f$ if and only if it has contact $\tau_{v}$ with $f^{-}$and $Q_{a(v)}^{v}$ vanish on $\varphi$. Hence, for any contact $\tau$ greater than $\tau_{v}$, the set $\mathcal{X}_{\tau, \ell}(\mathbf{g})$ is a disjoint union of piecewise affine bundles on $X_{0}(\mathbf{g}) \times \mathbb{G}_{m} \times B_{v}$ and the function $\operatorname{ac}(f \circ \mathbf{g})$ is given by the composition of the canonical map with the projection $X_{0}(\mathrm{~g}) \times \mathbb{G}_{m} \times B_{v} \longrightarrow \mathbb{G}_{m}$.

We can compute the difference $Z_{\geq v}-Z_{>v}^{-}$and check that it has limit $\Psi_{Q_{v}}\left(A_{v}\right)$ as $T$ goes to infinity.

It is a consequence of the following lemma, which follows from direct computation, that only extended rupture vertices have a non zero contribution.

4.4. Lemma. Consider a vertex $v$ of $\tau(f, p)$ and assume it is not an extended rupture vertex. Then the polynomial $Q_{v}$ is of the form: $Q_{v}(c, \omega)=\left(\left(c-\alpha \omega^{R}\right) \omega^{N}\right)^{E}$ where $R$, $N$ and $E$ are integers and $\alpha$ a non-zero constant. It defines a map from $\mathbb{A}_{k}^{1} \times \mathbb{G}_{m}$ to $\mathbb{A}_{k}^{1}$ the zero set of which is isomorphic to $\mathbb{G}_{m}$. Then:

- $\Psi_{Q_{v}}\left(A_{v}\right)=0$ in $\mathcal{M}_{X_{0}(\mathbf{g}) \times \mathbb{G}_{m}}^{\mathbb{G}_{m}}$.

- For the unique successor $s(v)$ of $v$, the equality

$$
A_{s(v)}=A_{v}
$$

holds in $\mathcal{M}_{X_{0}(\mathbf{g}) \times \mathbb{A}_{k}^{1} \times \mathbb{G}_{m}}^{\mathbb{G}_{m}}$. 


\section{References}

1. J. Denef and F. Loeser, Motivic Igusa zeta functions, J. Algebraic Geom. 7 (1998), 505-537.

2. $\ldots$, Germs of arcs on singular algebraic varieties and motivic integration, Invent. Math. 135 (1999), 201-232.

3. - Geometry on arc spaces of algebraic varieties, Proceedings of 3rd European Congress of Mathematics, Barcelona 2000, Progress in Mathematics 201 (2001), 327-348, Birkhaüser.

4. _ Lefschetz numbers of iterates of the monodromy and truncated arcs, Topology $4 \mathbf{1}$ (2002), 1031-1040.

5. H. Eggers, Polarinvarianten und die Topologie von Kurvensingularitäten, Dissertation, Rheinische Friedrich-Wilhelms-Universität, Bonn, 1982. Bonner Mathematische Schriften, 147. Universität Bonn, Mathematisches Institut, Bonn, 1982.

6. G. Guibert, Espaces d'arcs et invariants d'Alexander, Comment. Math. Helv. 77 (2002), $783-$ 820.

7. G. Guibert, F. Loeser, and M. Merle, Iterated vanishing cycles, convolution, and a motivic analogue of a conjecture of Steenbrink, Duke Math. J. 132 (2006), 409-457.

8. N Nearby cycles and composition with a non-degenerate polynomial, Int. Math. Res. Not. 31 (2005), 1873-1888

9. T. C. Kuo and Y. C. Lu, On analytic function germs of two complex variables, Topology 16 (1977), 299-310

10. E. Looijenga, Motivic Measures, Astérisque 276 (2002), 267-297, Séminaire Bourbaki, exposé 874 .

11. A. Némethi, The Milnor fiber and the zeta function of the singularities of type $f=P(h, g)$, Compositio Math. 79 (1991), 63-97.

12. __ Generalized local and global Sebastiani-Thom type theorems, Compositio Math. 80 (1991), 1-14.

13. — The zeta function of singularities, J. Algebraic Geom. 2 (1993), 1-23.

14. A. Némethi and J. Steenbrink, Spectral pairs, mixed Hodge modules, and series of plane curve singularities, New York J. Math. 1 (1994/95), 149-177.

15. M. Saito, On Steenbrink's conjecture. Math. Ann. 289 (1991), 703-716.

16. J. Steenbrink, Mixed Hodge structures on the vanishing cohomology, in Real and Complex Singularities, Sijthoff and Noordhoff, Alphen aan den Rijn, 1977, 525-563.

17. _ The spectrum of hypersurface singularities, in Théorie de Hodge, Luminy 1987, Astérisque, 179 -180 (1989), 163-184.

18. A. Varchenko, Asymptotic Hodge structure in the vanishing cohomology, Math. USSR Izvestija 18 (1982), 469-512.

15 Rue du Cap, 94000 Créteil, France

E-mail address: gilguibert@yahoo.fr

École Normale Supérieure, Département de mathématiques et applications, 45 rue D'Ulm, 75230 Paris Cedex 05, France (UMR 8553 du CNRS)

E-mail address: Francois.Loeser@ens.fr

URL: http://www.dma.ens.fr/ loeser/

Laboratoire J.-A. Dieudonné, Université de Nice - Sophia Antipolis, Parc Valrose, 06108 Nice Cedex 02, France (UMR 6621 du CNRS)

E-mail address: Michel.Merle@unice.fr

$U R L:$ http://www-math.unice.fr/membres/merle.html 